\title{
The Diabetic Foot: New Challenges in Management and Care
}

\author{
Nikolaos Papanas, ${ }^{1 *}$ Stella Papachristou ${ }^{2}$ \\ ${ }^{1}$ Internal Medicine-Diabetes Mellitus, Democritus University of Thrace, Greece \\ ${ }^{2}$ Laboratory of Toxicology, University of Crete, Greece
}

\section{Mini Review}

Diabetic foot ulcers (DFUs) remain difficult to heal and notoriously tend to relapse, approximately $40 \%$ at 1 year and $65 \%$ at 5 years. ${ }^{1}$ In this context, progress is needed in 6 areas:

\section{Improved early diagnosis of neuropathy}

Early diagnosis of diabetic polyneuropathy (DPN) is important for several reasons. ${ }^{1,2}$ First, careful and regular medical follow-up should be offered to avoid neuropathic DFUs. ${ }^{1,2}$ Secondly, optimised glycaemic control and correction of other vascular risk factors (e.g. hypertension, dyslipidaemia etc) should be diligently pursued., ${ }^{1,2}$ Finally, daily foot hygiene and appropriate footwear are indispensable. ${ }^{1}$ A number of practical bedside clinical tools contribute to improved early diagnosis, such as the indicator test Neuropad assessing sweat production in the feet, Vibra Tip, the portable NC-stat DPN Check device and others. ${ }^{2}$ Neuropad has been extensively studied and confirmed as an excellent, highly reproducible and practical screening tool with very high sensitivity and negative predictive value for DPN, which renders itself even for patient self-examination. ${ }^{2}$ Vibra Tip is a pocket-sized portable device which measures vibration perception at the hallux, whose diagnostic utility has recently been confirmed. ${ }^{3}$ NC-stat DPN Check is a special device for automated nerve conduction study of the sural nerve, which may be used by all health care professional after minimal training. ${ }^{2}$ We have shown that it yields very high diagnostic performance (sensitivity, specificity, positive and negative predictive value) in both diabetes types. ${ }^{4,5}$

\section{Improved early identification of patients at high risk}

Dryness of foot skin, as assessed by the indicator test Neuropad, has very recently been identified as an independent predictor of foot ulceration at 5 years. ${ }^{6}$ To this important purpose, the test yielded high sensitivity (86\%) but low specificity (49\%). ${ }^{6}$ Wider utilisation of this new screening tool is expected and should be encouraged.

\section{Improved detection and appreciation of Ischaemia}

Peripheral arterial disease (PAD) is common and may be formidable in diabetes mellitus ${ }^{7}$. Ankle brachial index (ABI) is widely employed, yet it may not detect distal but clinically relevant ischaemia. The latter, as demonstrated in a recent study, may more reliably be identified by the toe brachial index (TBI) in subjects with DFUs. ${ }^{7}$ Using arterial waveforms as a reference method, TBI had a higher AUC (area under the curve of the receiver operating characteristic curve) than $A B I$, suggesting that it can detect $P A D$ even if $A B I$ is normal. ${ }^{7}$ These findings may prove useful and lead to change of diagnostic work-up and guidelines.

\section{Improved organisation of diabetic foot clinics}

Modern diabetic foot clinics need to be re-organised to cope with the increasing burden of DFUs. ${ }^{8}$ Expert multidisciplinary care needs to be offered more quickly, especially in complex situations or multi-morbid patients. ${ }^{8}$ Timely debridement and administration of broad spectrum antibiotics, as well as urgent correction of ischaemia when needed, are of paramount importance in this endeavour. ${ }^{9,10}$ Limb-threatening Ischaemia and extensive infection with gangrene represent real emergencies, for which care should be offered as quickly as for stroke or myocardial infarction. ${ }^{10}$ This holds especially true for subjects with end-stage renal disease, in whom foot outcomes are, generally, more sinister. ${ }^{11}$

\section{Improved use of new/adjunctive modalities}

New therapeutic modalities are still being sought. Among these, hyperbaric oxygen therapy (both systemic and, more recently, lo-
Quick Response Code:

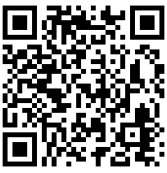

*Corresponding author: Nikolaos Papanas, Internal Medicine-Diabetes Mellitus, Democritus University of Thrace, Greece

Received: 08 September, 2020

Published: 25 September, 2020

Citation: Nikolaos P, Stella P. The Diabetic Foot: New Challenges in Management and Care. SOJ Cardio Curr Trend Surg. 2020;1(1):1-3. DOI: 10.53902/SOJCCTS.2020.01.000501 
cal) may improve wound healing in selected subjects with ischaemia. ${ }^{12-14}$ Improving nutrition is also thought to promote healing of DFUs. ${ }^{15} \mathrm{~A}$ well-balanced diet and healthy food habits under dietetic consultation contribute to improve healing. ${ }^{15}$

Another issue relates to chronic administration route for antibiotics. Intravenous use is less practical and may require prolonged hospitalisation, increasing health costs. A recent trial has shown that switch to oral antibiotics after a brief initial intravenous therapy is equally efficacious as long-term intravenous administration. ${ }^{16}$

In terms of wound care, several materials are considered as dressings for DFUs. One of these is sucrose octasulfate dressing. ${ }^{17}$ This has been shown safe and superior to standard wound care in healing of neuroischaemic DFUs at 20 weeks. ${ }^{17}$

A more recent approach is the use of adipose-derived mesenchymal cells (AMSCs) to enhance angiogenesis in subjects with diabetes and PAD. ${ }^{18}$ Experimental evidence suggests that AMSCs enhance wound healing, accelerate granulation tissue formation and increase re-epithelialisation and neovascularisation. However, clinical trials are needed before its efficacy can be fully delineated. ${ }^{18}$

\section{COVID-19 and its effects on diabetic foot care}

In the face of the new COVID-19 pandemic, a shift towards outpatient consultation and therapy emerged during the lockdown. ${ }^{19-21}$ Thus, a careful quick and reliable triage utilising telemedicine became necessary. ${ }^{19-21}$ In Italy, ${ }^{19} \mathrm{USA}^{20}$ and $\mathrm{UK}^{20}$ this has been shown to be feasible and achieve very satisfactory outcomes. Hospitalisation began to be reserved for complicated ulcers (severe infection, ischaemia, deep to bone ulcers etc). ${ }^{19,20}$ In hospitalised patients, treatment strategy should be determined by the urgency of surgical intervention and the complexity of DFUs. ${ }^{20-22}$ To help re-organisation of foot care and to standardise procedures, treatment algorithms have already been proposed. ${ }^{22,23}$ These appear useful and need further utilisation in expert centres.

\section{Conclusion}

DFUs still represent a daily therapeutic challenge. ${ }^{1}$ Progress is needed to improve diagnostic and therapeutic priorities. ${ }^{2}$ The situation is aggravated in the COVID-19 era, ${ }^{21}$ which has made telemedicine indispensable ${ }^{20}$ and emphasised the need for urgent and well-co-ordinated care.

\section{Acknowledgments}

None.

\section{Funding}

None.

\section{Conflicts of interest}

Author declares that there is no conflict of interest.

\section{References}

1. Armstrong DG, Boulton AJM, Bus SA. Diabetic foot ulcers and their recurrence. $N$ Engl J Med. 2017;376:2367-2375.
2. Papanas N. Diabetic neuropathy collection: progress in diagnosis and screening. Diabetes Ther. 2020;11:761-764.

3. Papanas N, Pafili K, Demetriou M. The diagnostic utility of vibratip for distal symmetrical polyneuropathy in type 2 diabetes mellitus. Diabetes Ther. 2020;11(1):341-346.

4. Papanas N, Chatzikosma G, Pafili K, et al. Evaluation of sural nerve automated nerve conduction study in the diagnosis of peripheral neuropathy in patients with type 2 diabetes mellitus. Arch Med Sci. 2016;12(2):390-393.

5. Papanas N, Pafili K, Demetriou M, et al. Automated measurement of sural nerve conduction is a useful screening tool for peripheral neuropathy in type 1 diabetes mellitus. Rev Diabet Stud. 2019;15:58-59.

6. Panagoulias G, Eleftheriadou I, Papanas N. Dryness of foot skin assessed by the visual indicator test and risk of diabetic foot ulceration: A prospective observational study. Frontiers Endocrinol. 2020.

7. Manu CA, Freedman B, Rashid H, et al. Peripheral arterial disease located in the feet of patients with diabetes and foot ulceration demands a new approach to the assessment of ischemia. Int J Low Extrem Wounds. 2020.

8. Huang DY, Wilkins CJ, Evans DR. The diabetic foot: the importance of coordinated care. Semin Intervent Radiol. 2014;31(4):307-312.

9. Manas $\mathrm{AB}$, Taori $\mathrm{S}$, Ahluwalia R.Admission time deep swab specimens compared with surgical bone sampling in hospitalized individuals with diabetic foot osteomyelitis and soft tissue infection. Int J Low Extrem Wounds. 2020.

10. Vas PRJ, Edmonds M, Kavarthapu V. The diabetic foot attack: Tis too late to retreat. Int J Low Extrem Wounds. 2018;17(1):7-13.

11. Sharma A, Vas P, Cohen S. Clinical features and burden of new onset diabetic foot ulcers post simultaneous pancreas kidney transplantation and kidney only transplantation. I Diabetes Complications. 2019;3(9):662-667.

12. Vas PR, Papanas N. Editorial and mini-review: topical oxygen therapy for diabetic foot ulcerations-avenue towards new hope?. Rev Diabet Stud. 2019;15:71-73.

13. Frykberg RG, Franks PJ, Edmonds M. A multinational, multicenter, randomized, double-blinded, placebo-controlled trial to evaluate the efficacy of cyclical topical wound oxygen (TWO2) therapy in the treatment of chronic diabetic foot ulcers: The TWO2 study. Diabetes Care. 2020;43(3):616-624.

14. Perren S, Gatt A, Papanas N, et al. Hyperbaric oxygen therapy in ischaemic foot ulcers in type 2 diabetes: a clinical trial. Open Cardiovasc Med J. 2018;12:80-85.

15. Vas PRJ, Edmonds ME, Papanas N. Nutritional supplementation for diabetic foot ulcers: the big challenge. Int J Low Extrem Wounds. 2017; 16:226-229.

16. Scarborough M, Li HK, Rombach I, et al. Oral versus intravenous antibiotics for bone and joint infections: the OVIVA non-inferiority RCT. Health Technol Assess. 2019;23:1-92.

17. Edmonds M, Lázaro-Martínez JL, Alfayate-García JM. Sucrose octasulfate dressing versus control dressing in patients with neuroischaemic diabetic foot ulcers (Explorer): an international, multicentre, doubleblind, randomised, controlled trial. Lancet Diabetes Endocrinol. 2018;6(3):186-196.

18. ÁlvaroAfonso FJ, SanzCorbalán I, Papanas N, et al. Adiposederived mesenchymal stem cells in the treatment of diabetic foot ulcers: a review of preclinical and clinical studies. Angiology. 2020;71(9):853-863.

19. Meloni M, Izzo V, Giurato L, et al. Management of diabetic persons with foot ulceration during COVID-19 health care emergency: Effectiveness of a new triage pathway. Diabetes Res Clin Pract. 2020;165:108-245. 
20. Shin L, Bowling FL, Armstrong DG, et al. Saving the diabetic foot during the COVID-19 pandemic: a tale of two cities. Diabetes Care. 2020;43(10):1704-1709.

21. Papanas N, Papachristou S. COVID-19 and diabetic foot: will the lamp burn bright?. Int J Low Extrem Wounds. 2020;19(2):111.

22. Kelahmetoglu O, Caml, MF, Kirazoglu A. Recommendations for management of diabetic foot ulcers during COVID19 outbreak. Int Wound J. 2020;17(5):1-4.
23. Meloni M, Bouillet B, Ahluwalia R. Fast-track pathway for diabetic foot ulceration during COVID-19 crisis: A document from International Diabetic Foot Care Group and D-Foot International. Diabetes Metab ResRev. 2020. 